\title{
Beurteilung von moderatem Weinkonsum und Alkoholmissbrauch aus Sicht deutscher und ungarischer Konsumenten
}

\author{
G. Szolnoki ${ }^{1}$, C. Stein-Hammer ${ }^{2}$, and D. Brazsil ${ }^{3}$ \\ ${ }^{1}$ Hochschule Geisenheim University, Institut für Wein- und Getränkewirtschaft, Professur für Marktforschung, Von-Lade-Str. 1, \\ 65366 Geisenheim, Germany \\ ${ }^{2}$ Deutsche Weinakademie, Platz des Weines 2, 55294 Bodenheim, Germany \\ ${ }^{3}$ National Council of the Wine Communities (HNT), Budafoki út 111, 1117 Budapest, Hungary
}

\begin{abstract}
Die Differenzierung zwischen moderatem Konsum alkoholischer Getränke und Alkoholmissbrauch wird in der Medizin, Soziologie und Politik zunehmend kontrovers diskutiert. Trotz sich zum Teil widersprechender Studienergebnisse, steht aktuell die Forderung im Raum nach generell niedrigeren Richtwerten für Alkohol, als in vielen Ländern festgelegt. Allerdings reicht die Spannbreite der weltweiten Guidelines von 10 bis 40 Gramm Alkohol/Tag. Für Deutschland werden je nach Absender 12 bzw. 14 Gramm täglich für Frauen und das Doppelte für Männer als moderat definiert, in Ungarn 17 Gramm (Frauen) und 34 Gramm (Männer). Dies wirft die Frage auf, wie Konsumenten den Zusammenhang zwischen Gesundheit, Weinkonsum und Alkoholmissbrauch einschätzen. Um auch kulturelle Unterschiede analysieren zu können, wurde 2017/18 eine repräsentative Befragung sowohl in Deutschland als auch in Ungarn mit 2000 bzw. 1500 Befragten durchgeführt. Die Befragungen beinhalteten Themen wie die Beurteilung und Definition des Alkoholmissbrauchs und moderaten Weinkonsums. Die Ergebnisse zeigen, dass in Ungarn der "moderate Weinkonsum" anders definiert wird als in Deutschland. Während die Deutschen die moderate Weinmenge durchschnittlich mit 0.311 Wein/Tag definieren, liegt der gleiche Wert in Ungarn bei 0.361. Auch im Fall des Alkoholmissbrauchs ergaben sich Unterschiede: Deutschland gibt dafür $0.761 \mathrm{Wein} /$ Tag und Ungarn 11 Wein/Tag an. Jedoch lassen sich auch Ähnlichkeiten, vor allem in der generellen Einschätzung von Alkoholmissbrauch erkennen. Unabhängig vom kulturellen Hintergrund waren die Befragten einig, dass übermäßiger Weinkonsum der Gesundheit schadet und dass sich nur leichter bis moderater Weinkonsum mit gesundem Lebensstil vereinbaren lässt.
\end{abstract}

\section{Einleitung und methode}

Der Konsum von alkoholhaltigen Getränken gehört für viele Menschen zum Alltag und ist in deren tägliches Leben integriert, sei es ein "Gläschen" Wein zum Essen oder ein "Bier am Abend" mit Freunden. Doch wie viel Alkohol ist gut für den Körper und ab wann beginnt der riskante oder gar schädliche Konsum? Was versteht man unter moderatem, mäßigem Konsum? Inwieweit ist der moderate Weinkonsum Teil eines Lebensstils, einer Weinkultur? Gibt es geschlechterspezifische Sichtweisen? Finden sich Unterschiede zwischen ungarischen und deutschen Konsumenten?

Die Weinwirtschaft klärt seit gut 10 Jahren mit ihrem Präventionsprogramm Wine in Moderation zum Beispiel in Schulen und Universitäten mit weinaffiner Ausbildung über gesundheitliche Aspekte des Weinkonsums auf, über seine positiven Aspekte und Risiken aber auch über die kulturellen und geschichtlichen Hintergründe des Genussgutes. Damit sollen bereits zu Beginn des Berufsweges die fachlichen aber auch persönlichen Kompetenzen der künftigen Weinwelt hinsichtlich dieser ambivalenten Thematik erhöht werden. Die angehende Branche wirkt hierbei als Multiplikator sowohl in Richtung
Konsument als auch in die eigene Branche (Stein-Hammer, 2014).

Wie weit sich dies gegebenenfalls schon zeigt, sollen die vorliegenden Repräsentativbefragungen aus Deutschland und Ungarn nachgehen.

\section{Hintergrund und überblick}

Gesundheitliche Effekte alkoholischer Getränke - und in diesem Kontext die Differenzierung zwischen moderatem Konsum und Alkoholmissbrauch - werden in der Medizin, Soziologie und Politik zunehmend kontrovers diskutiert. Einige Kompetenzen negieren die positiven Aspekte auch kleiner Mengen an Alkohol zunehmend und fordern generell niedrigere Richtwerte für Alkohol, als in vielen Ländern festgelegt (Wood, 2018; WHO, 2010).

Auch das Trinkmuster spielt eine zunehmend wichtige Rolle: Macht es doch einen Unterschied ob 30 Gramm Alkohol in Form von Wein zu einem (mediterranen) Essen genossen werden oder in Form von Spirituosen ohne Mahlzeit (Boban, 2016; Gea, 2014).

Obwohl Länder mit hohem BIP generell einen höheren Alkoholkonsum aufweisen, heißt es nicht, dass in diesen Ländern die meisten alkoholbezogenen Schäden und Hoch-Risiko-Trinkgewohnheiten auftreten (WHO, 2014). 
So finden wir in einigen westeuropäischen Ländern höchste Konsumraten, aber ihre Alkohol-AttributableMortalität ist relativ niedrig.

In vielen osteuropäischen Staaten findet man ebenfalls höchste Konsumwerte, riskante Trinkgewohnheiten und hohe Werte an alkoholbezogener Mortalität und Morbidität (WHO, 2014).

\subsection{Definition des standarddrinks}

Die unterschiedlichen alkoholischen Getränke enthalten unterschiedliche Mengen an Alkohol. Zudem werden sie in verschiedenen Größen serviert und verkauft. Die Frage nach einem einheitlichen Standarddrink (SD) stellt sich immer mehr, um eine Vergleichbarkeit zu erreichen.

Nun hat aber jedes Land seinen eigenen Standarddrink definiert (Alkohol/Ethanol in Gramm) und in unterschiedliche Empfehlungen für schädliches Trinkverhalten - über die Anzahl von Standarddrinks pro Tag oder pro Woche hinaus VERB?.

Die WHO definiert "standard drink" als "A volume of beverage alcohol (e.g. a glass of wine, a can of beer, or a mixed drink containing distilled spirits) that contains approximately the same amounts (in grams) of ethanol regardless of the type of beverage" (WHO, 1994).

Der Standarddrink rangiert von 8 bis 20 Gramm Ethanol, wobei $10 \mathrm{~g}$ als das häufigste Maß gilt (IARD, 2018). Da kein internationaler Konsens bzgl. der TrinkLeitlinien (drinking guidelines) besteht, definieren einige Länder in nationalen Richtlinien, wo der risikolose/ risikoarme/empfehlenswerte Grenzbereich liegt.

In Übereinstimmung mit der WHO (WHO, 2001), beinhaltet der niedrige Risikobereich die limitierte Alkoholaufnahme und Trinkmuster, die einen Schaden für sich und andere unwahrscheinlich machen.

Wissenschaftliche Daten zeigen, dass das Risiko erkennbar steigt, wenn mehr als 2 Drinks pro Tag konsumiert werden.

Nach WHO gelten folgende Regeln:

- Nicht mehr als 2 Drinks pro Tag;

- Nicht mehr als 5-mal pro Woche;

- Keine alkoholischen Getränke

- beim Autofahren oder Führen von Maschinen,

- in Schwangerschaft und Stillzeit,

- gemeinsam mit bestimmten Medikamenten,

- bei Problemen mit Alkohol.

Dem Umstand, dass Männer und Frauen den Alkohol unterschiedlich schnell metabolisieren, wird in vielen Richtlinien Rechnung getragen. Die meisten - aber nicht alle - Empfehlungen sehen für Frauen 50 - 75\% der Menge für Männer als geeignet an.

\subsubsection{Trends ändern sich}

Zwischen 2013 und 2016 existiert ein Trend zu Veränderungen der Guidelines in verschiedenen Ländern in folgende Richtung:

- Niedrigere Empfehlungen für Frauen und Männer (g Alkohol/Tag);

- Verschiedene Grenzwerte für verschiedene Altersgruppen (Jugend, junge Erwachsene, Erwachsene über 64);
- Statt tägliche eher wöchentliche Grenzwerte (oder beides);

- Empfehlungen zur Abstinenz für 2 Tage die Woche;

- Definitionen für niedrigen, moderaten, riskanten Alkoholkonsum.

So vollzog Italien 2014 mit neuen Richtlinien eine drastische Wende im Gegensatz zu denen von 2003. Ethanol wurde erstmals als toxische, krebserregende und psychoaktive Substanz beschrieben, für die es keine «Empfehlungen» gibt. Risikoarmer Konsum wurde als ein SD für Frauen und zwei SD für Männer festgelegt. (SINU, 2014)

Zudem gibt es keinen moderaten, risikofreien Alkoholkonsum, sondern nur Konsum mit niedrigem Risiko. Die Trinkrichtlinien verschieben sich von potentiell positiven Aspekten zu potentiellen Schäden. Diese neue Ansicht wird sich voraussichtlich in vielen Richtlinien niederschlagen.

\subsubsection{Deutschland und ungarn}

Der Pro-Kopf-Konsum an Alkohol liegt in Deutschland über 15-Jährige bei 11.81 der Bevölkerung und in Ungarn über 17-Jährigen bei 13,31 reiner Alkohol (WHO, 2014).

In Deutschland gelten nach der Bundeszentrale für Gesundheitliche Aufklärung BZGK $12 \mathrm{~g}$ (Frauen) und $24 \mathrm{~g}$ (Männer) am Tag als moderat. Zusätzlich werden zwei alkoholfreie Tage in der Woche empfohlen. Riskanter Konsum gilt ab 24 bis 60 g/Tag bzw. 12 bis 40 g/Tag, gefährlicher über $40 \mathrm{bzw}$. über $60 \mathrm{Gramm}$ Ethanol/ Tag. In Ungarn gelten 17 (Frauen) und 34 (Männer) Gramm Alkohol pro Tag als moderate Mengen.

Ein Standardrink wird in Deutschland mit 10-12 Gramm Ethanol, in Ungarn mit 17 Gramm definiert.

Schweres episodisches Trinken (mehr als $60 \mathrm{~g}$ in den letzten 7 Tagen) praktizieren in Deutschland $12.5 \%$ der Bevölkerung, in Ungarn 26.3\%.

Auch die Trinkmuster sind komplett unterschiedlich, was sicherlich auf die kulturellen und traditionellen Unterschiede zurückzuführen ist.

\subsubsection{Wine in moderation}

Als "moderat" kommunizieren die Leitlinien des Wine in Moderation-Programms bis zu 20 Gramm Alkohol (Größenordnung: $200 \mathrm{ml}$ Wein) pro Tag für die Frau und bis zu 30 Gramm für den Mann.

Dabei wird besonderen Wert auf gesunde Trinkmuster gelegt, wie z. B. Wein zur Mahlzeit und Wasser als Begleiter (WIM, 2016) und eingebunden in einen gesunden Lebensstil (Boban, 2016; Gea, 2014).

\subsection{Gesundheitliche aspekte}

Wein und Gesundheit ist ein häufig diskutiertes, ambivalentes Thema. Über Jahrhunderte hinweg wurde Wein als Naturheilmittel und Medikament verwendet. Wissenschaftlich mittlerweile unbestritten ist, dass die Wirkungen einer so genannte J-Kurve folgen: Demnach hat der geringe bis maßvolle Weinkonsum gesundheitlich positive Effekte, ab einer bestimmten Dosis aber ebenso unumstritten schädliche Folgen (Gaetano, 2017).

Als biologisch belegbar gilt z. B., dass geringer bis moderater Konsum protektive Effekte gegen folgende Krankheiten hat, wie 
- Herz-Kreislauf-Erkrankungen (Wood, 2018; Bell, 2017; Brien, 2011; Colpani, 2018; McCambridge, 2014; O'Keefe, 2014; Flesch, 2016),

- Diabetes mellitus Typ 2 (Hirst, 2016; Holst, 2017; Huang, 2017; Knott, 2015; Li, 2016),

- altersbedingte demenzielle Erkrankungen (Neafsey, 2011; Weyerer, 2011; Xu, 2017).

Bei höherem bis hohem Konsum treten schädigende Effekte auf alle Organsysteme auf, vor allem Risikoerhöhung für

- Krebserkrankungen (Xi, 2017; Schwingshackl, 2017; Kunzmann, 2018),

- Leberzirrhose (Rehm, 2010; Stein, 2016),

- Hirnblutung (Larsson, 2016; Costa, 2018).

Wein scheint dabei eine bessere Nutzen-Risiko-Bilanz zu haben als andere alkoholischen Getränke. Dies liegt zum einen an nicht-alkoholischen Inhaltsstoffen und zum anderen an den durchweg gesünderen Trinkmustern (Tresserra-Rimbau 2015; Estruch 2014, Schwingshackl 2017).

Für alkoholische Getränke über 1.2 Volumenprozent darf allerdings nicht mit gesundheitsbezogenen Angaben geworben werden (HCVO 2006).

\subsection{Gesellschaftliche aspekte}

Der Missbrauch alkoholischer Getränke jeder Art schadet der Gesundheit und zieht negative gesellschaftliche und volkswirtschaftliche Konsequenzen nach sich. Über die Zahl der Alkoholabhängigen in Deutschland gibt es unterschiedliche Angaben. Dies gründet zum Teil darin, dass "Abhängigkeit" unterschiedlich definiert ist.

Nach Terpe (2014) wurde z. B. im Jahr 2012 ein Anstieg der Alkoholkranken in Deutschland von ca. 36\% gegenüber 2006 festgestellt. Damit sei die Zahl der Alkoholkranken auf ca. 1,8 Millionen gestiegen.

Jährlich sterben ca. 15.000 Menschen in Deutschland an den Folgen zu hohen Alkoholkonsums. Zudem ging aus einer Erhebung des Instituts für Therapieforschung (München) hervor, dass noch immer zu viele Menschen unter 25 Jahren in riskanter Art und Weise Alkoholika konsumieren, was das Risiko einer manifesten Suchterkrankung im Erwachsenenalter erhöht (Tauner 2014).

Eine neue Studie zum Tabak- und Alkoholkonsum Jugendlicher in Deutschland (15.023 Teilnehmer) zeigt, dass gut die Hälfte der bis zu 17-Jährigen (51\%) schon einmal Alkohol getrunken hat, ein riskanter Alkoholkonsum wird von $12.1 \%$ praktiziert, von regelmäßigem Rauschtrinken berichten 7\%. Allerdings zeigt sich, dass sowohl der riskante Alkoholkonsum als auch das regelmäßige Rauschtrinken vom Erhebungszeitraum 2009-2012 zum Erhebungszeitraum 2014-2017 stark abnahmen. Das deutet auf Erfolge von Präventionsprogrammen in Deutschland hin (RKI, 2018).

Dass der sozioökonomische Status europaweit einen Effekt auf alkoholbezogene Schäden hat, ist nicht mehr zu bezweifeln. So weisen sozial benachteiligte Gruppen höhere alkoholbezogene Schäden auf als nicht benachteiligte - sowohl was die Anzahl der Drinks angeht als auch das ungesündere Trinkmuster, mehr Übergewicht und den Rauchstatus (Katikireddi, 2017).
Dabei ist die Bereitschaft zum Weinkonsum grundsätzlich nicht von soziodemografischen Faktoren abhängig. Man kann sagen, dass Wein in der Bevölkerung als Getränk sehr breit akzeptiert wird. Vergleicht man jedoch die Konsumintensität von Weintrinkern, wird es klar, dass Einkommen, Bildung oder Alter einen signifikanten Einfluss auf die konsumierte Menge nehmen (Szolnoki, Hoffmann, 2014).

Die soziale Komponente des Weingenusses darf nicht außen vorgelassen werden und hat wesentlichen Einfluss auch über den kurzfristigen Hedonismus hinaus (Haucap, 2014)

\section{Material und methode}

Die im zweiten Kapitel erwähnten Ergebnisse unterschiedlicher Studien werden weltweit kontrovers diskutiert. In dieser Untersuchung wird jedoch versucht, die Alkoholproblematik aus Sicht der Verbraucher $\mathrm{zu}$ analysieren und herauszufinden, wie deutsche und ungarische Weinkonsumenten den Zusammenhang zwischen Gesundheit, Weinkonsum und Alkoholmissbrauch einschätzen.

Die Datenerhebung erfolgte im Rahmen einer faceto-face Befragung in Deutschland mit 2.000 und in Ungarn mit 1.500 Probanden. Die Teilnehmer der Studie wurden nach repräsentativen Quoten ausgewählt, dementsprechend können wir davon ausgehen, dass die Ergebnisse die Meinung der Grundgesamtheit, d. h. der Bevölkerung der Bundesrepublik Deutschland und der Republik Ungarn widerspiegeln. Die Daten wurden 2018 im Rahmen von zwei Omibus-Befragungen eines professionellen Markforschungsunternehmens (GfK, Nürnberg) erhoben.

Der Fragebogen basierte auf der Studie von Szolnoki und Hoffmann (2014) und beinhaltete neben den spezifischen Fragen zum Thema moderater Weinkonsum und Alkoholmissbrauch auch soziodemographische und verhaltensbezogene Fragen.

Die Daten wurden mit der Software SPSS 23.0 (IBM) ausgewertet. Für die Auswertung kamen statistische Methoden wie Häufigkeit, Mittelwert, Kreuztabelle und ANOVA zum Einsatz.

\section{Ergebnisse und diskussion}

\subsection{Konsumhäufigkeit unterschiedlicher alkoholischen Getränke}

Zunächst wurde eine Untersuchung der Konsumhäufigkeit bei drei alkoholischen Getränken durchgeführt, um Verhaltensunterschiede zwischen deutschen und ungarischen Verbrauchern zu ermitteln. Tabelle 1 zeigt, dass zwischen den beiden Ländern erhebliche Unterschiede in Bezug auf den Verbrauch von Stillwein, Schaumwein und Bier bestehen. Der Vergleich zeigt auch, dass die Gruppe der Häufig-Weintrinker (diese Kategorie fasst Konsumenten der ersten zwei Häufigkeitskategorien zusammen) in Ungarn deutlich größer ist als in Deutschland (22 Prozent gegenüber 14 Prozent). Demnach ist der Anteil der Gelegentlich-Trinker und Nicht-Weintrinker in Deutschland höher als in Ungarn.

Mit einem Pro-Kopf-Wert von 3.41 ist Deutschland weltweit die Nummer eins beim Sektverbrauch 
Tabelle 1. Vergleich der Konsumhäufigkeit bei Stillwein, Schaumwein und Bier.

\begin{tabular}{|c|c|c|c|c|c|c|}
\hline & \multicolumn{2}{|c|}{ Stillwein } & \multicolumn{2}{|c|}{ Schaum-wein } & \multicolumn{2}{|c|}{ Bier } \\
\hline & $\begin{array}{c}\text { GER } \\
n=2000\end{array}$ & $\begin{array}{c}\text { HUN } \\
n=1500\end{array}$ & $\begin{array}{c}\text { GER } \\
n=2000\end{array}$ & $\begin{array}{c}\text { HUN } \\
n=1500\end{array}$ & $\begin{array}{c}\text { GER } \\
n=2000\end{array}$ & $\begin{array}{c}\text { HUN } \\
n=1500\end{array}$ \\
\hline Mehrmals/Woche & $6 \%$ & $11 \%$ & $1 \%$ & $0 \%$ & $28 \%$ & $13 \%$ \\
\hline Einmal/Woche & $8 \%$ & $11 \%$ & $3 \%$ & $1 \%$ & $17 \%$ & $11 \%$ \\
\hline 2-3 Mal/Monat & $12 \%$ & $14 \%$ & $9 \%$ & $3 \%$ & $11 \%$ & $15 \%$ \\
\hline Einmal/Monat & $10 \%$ & $12 \%$ & $12 \%$ & $6 \%$ & $6 \%$ & $8 \%$ \\
\hline Weniger als $1 \mathrm{Mal} / \mathrm{Monat}$ & $24 \%$ & $19 \%$ & $49 \%$ & $54 \%$ & $13 \%$ & $19 \%$ \\
\hline Nie & $39 \%$ & $34 \%$ & $27 \%$ & $37 \%$ & $25 \%$ & $35 \%$ \\
\hline Chi-Quadrat & \multicolumn{2}{|c|}{$68.421 *$} & \multicolumn{2}{|c|}{$144.739 *$} & \multicolumn{2}{|c|}{$177.311^{*}$} \\
\hline Cramer-V & \multicolumn{2}{|c|}{0.198} & \multicolumn{2}{|c|}{0.284} & \multicolumn{2}{|c|}{0.302} \\
\hline
\end{tabular}

Tabelle 2. Einschätzung des moderaten Weinkonsums pro Tag (13 Vol. \% Alk. Wein) in Liter und Gramm reinem Alkohol.

\begin{tabular}{|l|c|c|c|}
\hline & Weintrinker & $\begin{array}{c}\text { Nicht- } \\
\text { Weintrinker }\end{array}$ & $\begin{array}{c}\text { Alle } \\
\text { Befragten }\end{array}$ \\
\hline Deutschland & $0.341(35.4 \mathrm{~g})$ & $0.271(28.1 \mathrm{~g})$ & $0.311(32.4 \mathrm{~g})$ \\
\hline Ungarn & $0.381(39.5 \mathrm{~g})$ & $0.321(33.3 \mathrm{~g})$ & $0.361(37.4 \mathrm{~g})$ \\
\hline F-Wert & $23.179^{*}$ & $11.003^{*}$ & $48.992^{*}$ \\
\hline Sign. & 0.000 & 0.001 & 0.000 \\
\hline
\end{tabular}

(DWI, 2018). Dies zeigt auch das Ergebnis in Tabelle 1 an, insbesondere wenn die ersten Häufigkeitskategorien verglichen werden. In Ungarn ist Sekt ebenfalls beliebt, die Trinkhäufigkeit ist jedoch deutlich niedriger als in Deutschland.

Der Bierkonsum in Deutschland übersteigt den in Ungarn (DWI, 2018; HNT, 2016). Wenn die ersten beiden Kategorien die Häufig-Biertrinker darstellen, sieht man, dass 45 Prozent der deutschen Bevölkerung mindestens einmal pro Woche Bier trinken. In Ungarn beträgt dieser Anteil lediglich 24 Prozent.

\subsection{Moderater und übermäßiger weinkonsum}

Ziel der Untersuchung war zum einen herauszufinden, wie deutsche und ungarische Konsumenten moderaten bzw. übermäßigen Weinkonsum definieren. Dafür wurden zwei Selbsteinschätzungsfragen gestellt: "Bis $\mathrm{zu}$ wie vielen Gläsern Wein/Tag halten Sie Weinkonsum für moderat?" und "Ab wie vielen Gläsern Wein/Tag handelt es sich um übermäßigen Weinkonsum?". Mit der Frage wurde gleichzeitig auch die Größe des Glases definiert - ein Glas $200 \mathrm{ml}$ mit einem Wein von 13 Vol. \% Alk.

Wie Tabelle 2 zeigt, unterscheiden sich die Ergebnisse bezüglich des moderaten Konsums sowohl zwischen den Ländern als auch zwischen Weintrinkern und Nicht-Weintrinkern signifikant. Generell kann festgestellt werden, dass die Ungarn eine höhere Grenze sowohl bei moderatem als auch bei übermäßigem Alkoholkonsum gesetzt haben als die Deutschen. Der moderate Weinkonsum wird in Deutschland mit 0.31 Wein/Tag definiert. Dies entspricht 32.2 Gramm reinem Alkohol. In Ungarn hingegen sind es 0.361 mit 37.4 Gramm reinem Alkohol. Die deutsche Hauptstelle für Suchtfragen gibt als Grenzwert für einen risikoarmen Konsum 12 Gramm reinen Alkohol für Frauen und 24 Gramm für Männer an, während andere Stellen nicht mehr Alkohol als 20 Gramm für Frauen und 30 Gramm für Männer raten. Die Werte der
Tabelle 3. Einschätzung des übermäßigen Weinkonsums pro Tag (13 Vol. \% Alk. Wein) in Liter und Gramm reiner Alkohol.

\begin{tabular}{|l|c|c|c|}
\hline & Weintrinker & $\begin{array}{c}\text { Nicht- } \\
\text { Weintrinker }\end{array}$ & $\begin{array}{c}\text { Alle } \\
\text { Befragten }\end{array}$ \\
\hline Deutschland & $0.801(83.2 \mathrm{~g})$ & $0.701(72.7 \mathrm{~g})$ & $0.761(79.0 \mathrm{~g})$ \\
\hline Ungarn & $1.101(114.4 \mathrm{~g})$ & $0.851(88.4 \mathrm{~g})$ & $1.01(104.0 \mathrm{~g})$ \\
\hline F-Wert & $23.179^{*}$ & $11.003^{*}$ & $240.959^{*}$ \\
\hline Sign. & 0.000 & 0.001 & 0.000 \\
\hline
\end{tabular}

Untersuchung sind außerhalb der empfohlenen Grenze. In Ungarn liegt die empfohlene Menge bei 17 Gramm (Frau) und 34 Gramm (Mann). Auch hier überschreiten die Werte der Einschätzungen die offiziellen Grenzen.

Beim Vergleich männlicher und weiblicher Befragten zeichnet sich, unabhängig vom Land, ein signifikanter Unterschied ab. Männer schätzen den moderaten Weinkonsum signifikant höher ein als Frauen. Vergleicht man jedoch die empfohlene Konsummenge, wird es klar, dass die Abweichung der Frauen von der empfohlenen Menge deutlich größer ist als die von Männern.

Die unterschiedlichen Altersgruppen haben den moderaten Weinkonsum ähnlich bewertet - allerdings die Ungarn auf einem signifikant höheren Level.

Übermäßiger Weinkonsum ist aus Sicht der Bevölkerung mehr als das zweifache der moderaten Konsummenge. Weintrinker in Deutschland schätzten den Wert des übermäßigen Weinkonsums auf 0.81 , während Nicht-Weintrinker 0.11 weniger angaben. In Ungarn liegen die Werte deutlich höher. Weintrinker gaben durchschnittlich 1.11 und Nicht-Weintrinker 0.851 als übermäßig an (Tabelle 3).

Alter scheint die Beurteilung des übermäßigen Weinkonsums nicht signifikant beeinflusst zu haben.

Zusammenfassend kann festgestellt werden, dass sowohl in der gesamten Stichprobe als auch in der Gruppe der Weintrinker die Werte eindeutig höher bewertet wurden als die offiziellen Werte.

\subsection{Imagebild der konsumenten über alkohol - und weinkonsum}

Um die Einstellung der Konsumenten zum Thema "Alkohol - und Weinkonsum" zu analysieren, wurden sechs Statements auf einer Skala von -3 (lehne ich voll und ganz ab) bis +3 (stimme ich voll und ganz zu) abgefragt. Fünf der Statements waren Wein- und eine Alkohol-bezogen. Beim Vergleich der deutschen und 


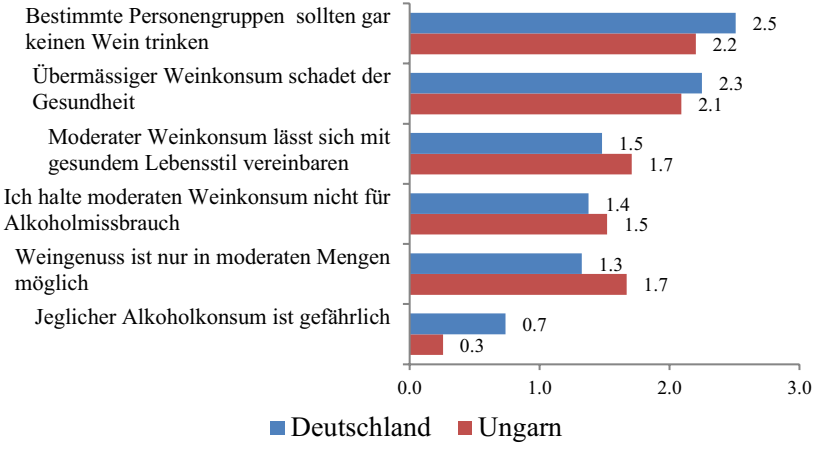

Abbildung 1. Vergleich der Aussagen zum Alkoholkonsum und Wein (gemessen auf einer Skala von -3 bis +3 ).

ungarischen Ergebnisse wird deutlich, dass Konsumenten aus beiden Ländern die Statements mehr oder weniger ähnlich bewerteten (siehe Abb. 1).

Sowohl die Deutschen als auch die Ungarn stimmen überein, dass bestimmte Personen (Schwangere, Jugendliche unter 16/18 Jahren) keinen Wein trinken sollten und dass übermäßiger Weinkonsum der Gesundheit schadet - diese Statements wurden in beiden Ländern im Vergleich zu den anderen Aussagen signifikant höher bewertet.

Die Fragen zum Thema "moderater Weinkonsum" verbunden mit "Lebensstil", "Weingenuss" und "Alkoholmissbrauch" wurden von beiden Nationen ähnlich beurteilt, wobei die ungarischen Konsumenten alle drei Statements etwas höher bewerteten als die Deutschen.

Das überspitzte Statement "jeglicher Alkoholkonsum ist gefährlich" wurde in Deutschland etwas höher bewertet, jedoch ist hier der Anteil von Nicht-Weintrinkern, die diesem Statement zugestimmt haben, sehr groß.

Vergleicht man die Weintrinker mit Nicht-Weintrinkern, wird es klar, dass die Beurteilung der Statements sehr stark von der persönlichen Erfahrung und dem Konsum von Wein abhängt. Nicht-Weintrinker aus beiden Ländern sind viel weniger mit den Aussagen wie "moderater Weinkonsum lässt sich mit gesundem Lebensstil verbinden", "ich halte moderaten Weinkonsum nicht für Alkoholmissbrauch" und "Weingenuss ist nur in moderaten Mengen möglich" einverstanden als Weintrinker. Überraschenderweise bewerten jedoch beide Gruppen die ersten beiden Aussagen ziemlich identisch, ohne signifikante Unterschiede. Dies signalisiert eine generelle Sensibilisierung der Bevölkerung unabhängig von Konsumintensität für kritische Fragen, wie gefährdete Zielgruppe und Alkoholkonsum sowie übermäßigen Weinkonsum und Gesundheit.

Auch das Geschlecht verursacht Unterschiede bei der Beurteilung der Aussagen. Demnach bewerten Frauen fast alle Statements signifikant unterschiedlicher als Männer. Dies zeugt von einer ausgeprägten Sensibilität der weiblichen Konsumenten im Gegensatz zu Männern bezüglich Alkohol und Gesundheit.

Der Altersunterschied scheint die Meinungen zum Thema Weinkonsum nicht stark $\mathrm{zu}$ beeinflussen. In Deutschland sticht lediglich die jüngste Generation (16-29 Jahre alt) mit ihrer eindeutig weicheren Meinung dem Alkohol- und Weinkonsum gegenüber heraus, während in Ungarn alle Altersklassen die Statements ähnlich beurteilten.
Häufig-Weintrinker, die mindestens einmal pro Woche Wein konsumieren, sind gegenüber den hier aufgelisteten Statements nachsichtiger als Gelegentlich- oder SeltenTrinker. Dieses Phänomen taucht sowohl in Deutschland als auch in Ungarn auf.

\section{Zusammenfassung}

Die hier dargestellte Studie hatte das Ziel, die Einschätzung von Konsumenten aus Deutschland und Ungarn zum moderatem Weinkonsum und Alkoholmissbrauch zu analysieren. Dafür wurde eine repräsentative Befragung mit 2.000 in Deutschland und 1.500 Befragten in Ungarn durchgeführt. Obwohl Wein generell das Image genießt, gesünder als andere alkoholische Getränke zu sein (Higgins und Llanos, 2015; Chang et al., 2016), haben Konsumenten eine klare Vorstellung, wo moderater Weinkonsum aufhört und wo übermäßiger Weinkonsum anfängt. Jedoch liegen die von den Konsumenten eingeschätzten Werte deutlich über den offiziell empfohlenen Grenzen. In Deutschland wird von Konsumenten moderater Konsum mit 32.4 Gramm und in Ungarn mit 37.4 Gramm reinem Alkohol/ Tag definiert. Auf die Frage "Wo fängt ein übermäßiger Weinkonsum pro Tag an", reagierten die Konsumenten noch nachsichtiger und gaben in Deutschland 79 Gramm und in Ungarn 104 Gramm reinen Alkohol/ Tag an.

Die Mehrheit der befragten Deutschen und Ungarn stimmen überein, dass gefährdete Zielgruppen wie Schwangere oder Minderjährige den Weinkonsum meiden sollten und dass zu viel Wein gesundheitsschädlich ist. Weitere Aussagen über moderaten Weinkonsum wurden je nach Geschlecht, Alter und Konsumhäufigkeit signifikant unterschiedlich beurteilt, jedoch ergaben sich keine große Unterschiede zwischen den deutschen und ungarischen Ergebnissen.

Die Befragten bewerteten die Gefährlichkeit jeglichen Alkoholkonsums moderater - diese Aussage erreichte einen Durchschnitt von 0.7 in Deutschland und 0.3 in Ungarn (gemessen auf einer Skala von -3 bis +3 ).

Die hier präsentierten Ergebnisse helfen zu verstehen, wie Konsumenten moderaten und übermäßigen Weinkonsum im Alltag empfinden und wie sie Wein als alkoholisches Getränk beurteilen. Mithilfe dieser Ergebnisse kann so die Zielgruppe der Häufig-Trinker genau beschrieben werden. Diese Erkenntnisse können zu einer langfristigen zielorientierten Wine in ModerationStrategie für Konsumenten beitragen und die politische Beratung zum Thema "Moderater und übermäßiger Weinkonsum" unterstützen.

\section{References}

[1] M.M. Bergmann, J. Rehm, K. Klipstein-Grobusch, et al., Int. J. Epidemiol. 42, 6 (2013)

[2] S. Bell, M. Daskalopoulou, E. Rapsomaniki, et al., BMJ 356, 909 (2017)

[3] M. Boban, Food Funct. 7, 2937 (2016)

[4] S.E. Brien, P.E. Ronksley, B.J. Turner, K.J. Mukamal, W.A. Ghali, BMJ 342, 636 (2011)

[5] BZGA2018, Alkoholwissen - Kompakt. https:// www.kenn-dein-limit.de/alkohol/ alkoholwissen-kompakt/ 
[6] K.J. Chang, M.L. Thach, J. Olsen, Wine Econ. Policy 5, 105 (2016)

[7] V. Colpani, C.P. Baena, L. Jaspers, et al., Eur. J. Epidemiol. 33, 9 (2018)

[8] P. Costa, M. Grassi, L. Iacoviello, et al., Neurology 91, 227 (2018)

[9] R. Estruch, R.M. Lamuela-Raventos, Nutrition Aging 2, 101 (2014)

[10] G. de Gaetano, S. Costanzo, J. Am. Coll. Cardiol. 70, 923 (2017) dx.doi.org/10.1016/j.jacc.2017 07710

[11] A. Gea, M. Bes-Rastrollo, E. Toledo, et al., Br. J. Nutr. 111, 10 (2014)

[12] Deutsches Weininstitut (DWI), Deutscher Wein Statistik 2017-2018. Deutsches Weininstitut, 2018, Bodenheim, Germany

[13] M. Flesch, et al., Cardiovascular Diseases (2016) ISSN 0340-9937. DOI 10.1007/s00059-016-4467-8

[14] J. Haucap, A. Herr, Eur. J. Law Econ. (2014) http://ssrn. com/abstract $=1548508$

[15] Health Claims Verordnung, VO EG Nr. 1924/2006 des Europäischen Parlaments und des Rates über nährwert- und gesundheitsbezogene Angaben über Lebensmittel

[16] Hegyközségek Nemzeti Tanácsa (HNT), Magyarország szőlészetének és borászatának helyzete. Unpublished document, 2016, Budapest, Hungary

[17] L.M. Higgins, E. Llanos, Wine Econ. Policy 15, 4 (2015)

[18] IARD 2018 Drinking Guidelines: General Population, http://www.iard.org/policy-ables/ drinking-guidelines-general-population/

[19] S. Katikireddi, et al., Socioeconomic status as an effect modifier of alcohol consumption and harm: analysis of linked cohort data, The Lancet Published Online May 10, 2017 http://dx.doi. org/10.1016/S2468-2667 (17) 30078-6

[20] A.T. Kunzmann, H.G. Coleman, W.Y. Huang, S.I. Berndt, PLoS Med. 15, e1002585 (2018)

[21] S.C. Larsson, A. Wallin, A. Wolk, H.S. Markus, BMC Med. 14, 178 (2016)

[22] J. McCambridge, et al., Drug \& Alcohol Rev. 34, 58 (2014)

[23] E.J. Neafsey, M.A. Collins, Neuropsychiatr. Dis. Treat. 11, 7 (2011)

[24] OECD2015: http://www.oecd.org/health/ health-systems/tackling-harmful-alcoholuse-9789264181069-en.htm

[25] J.H. O’Keefe, S.K. Bhatti, A. Bajwa, J.J. Dinicolantonio, C.J. Lavie, Mayo Clin. Proc. 89, 382 (2014)

[26] J. Rehm, B. Taylor, S. Mohapatra, et al., Drug Alcohol Rev. 29, 4 (2010)

[27] RKI 2018, J. Health Monitor. 3 (2018) DOI 19.17886/RKI-GBE-2018-066 Robert-Koch-Institut, Berlin

[28] Sassi, F. (ed.), Tackling Harmful Alcohol Use: Economics and Public Health Policy (OECD Publishing, Paris, 2015) http://dx.doi.org/ 10.1787/9789264181069-en
[29] L. Schwingshackl, C. Schwedhelm, C. Galbete, G. Hoffmann, Nutrients 9 (2017)

[30] SINU, 2014, LARN - Livelli di Assunzione di Riferimento di Nutrienti ed Energia per la popolazione italiana, Milano Societa Italiana di Nutrizione Umana (SINU)

[31] E. Stein, M. Cruz-Lemini, J. Altamirano, et al., J. Hepatol. 65, 5 (2016)

[32] C. Stein-Hammer, Das Deutsche Weinmagazin 25/26 (2014)

[33] G. Szolnoki, und D. Hoffmann, Neue Weinkundensegmentierung in Deutschland. Gesellschaft zur Förderung der Hochschule Geisenheim e. V., 2014, Germany

[34] G. Szolnoki, D. Hoffmann, Int. J. Wine Business Res. 26, 27 (2014) https://doi.org/10.1108/ I JWBR-10-2012-0028

[35] S. Tauner, Deutschland einig Trinkerland. Abgerufen am 25.01.17 von http://www.stern.de/ gesundheit/15-000-alkohol-tote-jaehrlichdeutschland-einig-trinkerland3165222.html (2014)

[36] H. Terpe, Deutliche Zunahme von Suchterkrankungen - Bundesregierung vernachlässigt Suchtkranke. Abgerufen am 25.01.17 von https://www.gruene-bundestag.de/presse/ pressemitteilungen/2014/januar/deutlichezunahme-von-suchterkrankungenbundesregierung-vernachlaessigtsuchtkranke.html (2014)

[37] A. Tresserra-Rimbau, A. Medina-Remon, R.M. Lamuela-Raventos, et al., Br. J. Nutr. 113, 121 (2015)

[38] S. Weyerer, M. Schaufele, B. Wiese, et al., Age Ageing 40, 456 (2011)

[39] W. Xu, H. Wang, Y. Wan, et al., Eur. J. Epidemiol. 32, 1 (2017)

[40] B. Xi, S.P. Veerank, et al., J. Am. Coll. Cardiol. 70, 913 (2017) dx.doi.org/10.1016/j.jacc.2017 06054.

[41] WHO, Brief Intervention; 2001. Available at: http: //apps.who.int/iris/bitstream/ handle/10665/67210/WHO_MSD_MSB_01.6b.pdf ; jsessionid=F5E89FF34B095802E3E66B38F8B47 $6 \mathrm{D} 6$ ? sequence $=1$

[42] WHO, Global strategy to reduce the harmful use of alcohol; 2010. Available at: http://www . who.int/substance_abuse/publications/ global_strategy_reduce_harmful_use alcohol/en/

[43] WHO, Global status report on alcohol and health; 2014. Available at: http://www.who.int/ substance_abuse/publications/global_ alcohol_report/msbgsruprofiles.pdf?ua=1)

[44] WineinModeration https://www.wineinmodera tion.eu/de/home/list

[45] A. Wood, et al., Risk thresholds for alcohol consumption: combined analysis of individualparticipant data for 599912 current drinkers in 83 prospective studies. www. thelancet.com, 391, 1513 (2018) 